\title{
APPLICATIONS OF LANGMUIR FILMS IN INTEGRATED OPTICS
}

\author{
C. W. PITT and L. M. WALPITA \\ University College London, Department of Electronic \& Electrical Engineering, Torrington Place, \\ London WC1E 7JE, England.
}

(Received December, 1976)

\begin{abstract}
This paper describes the preparation and properties of Langmuir (long-chain fatty-acid) films, for use as optical waveguides. It is shown how the refractive index and molecular thickness can be estimated by probing the films with a guided wave, and how such layers may be utilised to modify the propagation characteristics of pre-fabricated guiding structures. Cases of isotropic and anisotropic guides formed from such films are discussed, together with further uses of such films as cladding to guides and other materials.
\end{abstract}

\section{INTRODUCTION}

Integrated optics is a term often applied to the capability to manipulate optical signals in a thin dielectric film. The essential features of this type of device are a thin film of transparent material supported by a transparent substrate; the light is confined within the thin film by arranging for the film to have a higher refractive index than the substrate so that total internal reflection occurs within the film.

Assuming that the refractive index condition has been satisfied, then the optical waveguide must also meet certain thickness and optical loss requirements to be of practical use. The film thickness, usually of the order of the wavelength of the guided light, determines the interference between successive reflections from the film interfaces; constructive interference is required for energy to remain guided. The optical loss, which includes both scattering of light from inhomogeneities in the film and absorption of light, is extremely important in terms of the performance of an integrated optical device; for example in a thin film applied to a conventional optical component such as a lens, the light passes through the film only once - a thickness of a few thousand Angström, but in a waveguide the light travels along the plane of the film a distance of several millimetres, and perhaps centimetres.

Numerous techniques have been developed or modified for fabricating thin film waveguides including thermal evaporation in a vacuum ${ }^{1}$, sputtering $^{2}$, solution depostion ${ }^{3}$ and diffusion of dopants into single crystal substrates ${ }^{4}$. All of these techniques produce films which exhibit some advantages and several limitations depending on the particular application of the integrated optical component. In this paper we explore a technique for depositing organic thin films. This technique, often called the Langmuir-Blodgett process $5,14,15$ enables the dielectric film to be deposited by sequential stacking of monomolecular layers. The long chain fatty acids which compose the layers have a unique molecular length, which is known, in principle, to within a fraction of an Angström - so that the total film thickness may be precisely controlled by depositing the desired number of layers. The thickness control thus achieved is considerably better than that obtained by any other technique. We will also indicate that the refractive index may be altered, again in a very precise manner, enabling waveguides to be designed and fabricated with defined propagation characteristics.

The Langmuir-Blodgett technique is conceptually simple - a small quantity of the fatty-acid is dispersed on a water surface, compressed to form a continuous monolayer, and is then removed onto a substrate by immersing the substrate through the monolayer. A section of the organic film adheres to the substrate on each pass through the monolayer, increasing the film thickness by a monolayer ( 25 to $50 \AA$ ) for each immersion.

However, before discussing the deposition process and the results of tests on Langmuir film waveguides, we present a brief resumé of the principles of optical waveguiding. 


\section{BASIC OPTICAL WAVEGUIDE THEORY}

In order to obtain optical guiding in dielectric films, it is necessary that the film and the substrate are both optically transparent. Figure 1 shows a crosssection of a planar waveguide; for simplicity the entire structure is considered to be isotropic. Optical guiding is achieved in this structure by total internal reflection of the plane waves comprising the light in

\section{$n_{\text {superstrate }}\left(n_{2}\right)$}

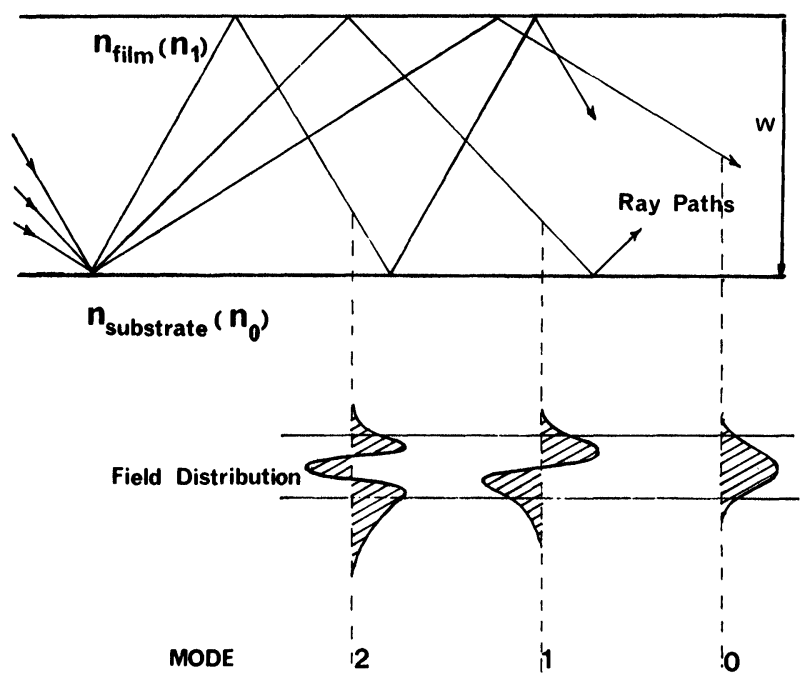

FIGURE 1 Diagram of optical paths in an isotropic planar waveguide.

the film. A single ray path, as indicated, corresponds to a particular electric field distribution in the guide. The initial condition for guiding is given by:

$$
n_{1}>n_{2}, n_{0}
$$

where $n_{0}, n_{1}, n_{2}$ are the refractive indices of the substrate, film and the superstrate. To gain insight into wave propagation in the film, we define the wave vector (i.e. the velocity of the ray in the film material) in the ray direction as $k n_{1}$ where $k=2 \pi / \lambda$, the free space wave vector. This wave vector may then be resolved into two components, see Figure 2:

$$
b_{m}=k n_{1} \cos \theta_{m} \quad \text { and } \quad \beta_{m}=k n_{1} \sin \theta_{m}
$$

In order for the optical power to flow in the $x$ direction, the incident wave front and the wavefront which is totally reflected at each of the two boundaries must interfere constructively; that is, the round
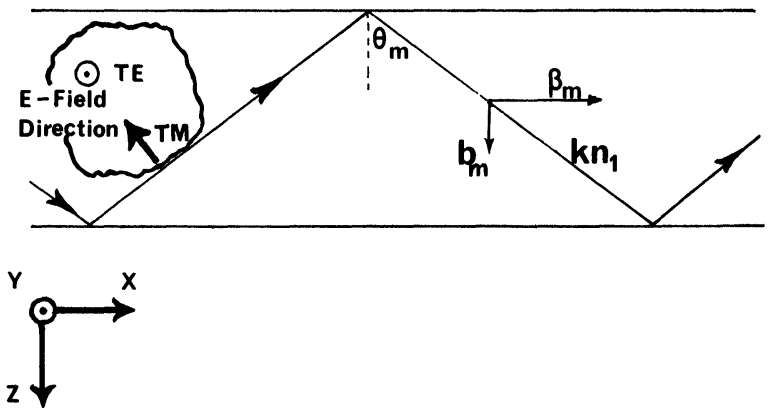

FIGURE 2 Diagram showing the resolution of the wave vector in a planar waveguide, into two components.

trip phase change of the wave component in the $z$ direction must be $2 m \pi$, where ' $m$ ' is an integer. This condition can be formally stated ${ }^{6}$ as:

$$
\begin{aligned}
& 2 b_{m} w+2\left(\phi_{10}+\phi_{12}\right) \\
& {\left[\begin{array}{l}
\text { The phase change due } \\
\text { to the double passage } \\
\text { of the ray } z \text {-compo- } \\
\text { nent through the } \\
\text { thickness of the film }
\end{array}\right]+\left[\begin{array}{l}
\phi_{10}, \phi_{12} \text { phase changes } \\
\text { due to the ray being } \\
\text { reflected at the film } \\
\text { substrate and film-air } \\
\text { boundaries }
\end{array}\right]} \\
& =2 m \pi \\
& =\left[\begin{array}{l}
\text { ' } m \text { ' the integer } \\
\text { is called the } \\
\text { "mode number" }
\end{array}\right]
\end{aligned}
$$

where the Fresnel reflection expression defines the phase change at the interfaces:

$$
\tan \phi_{i j m}=A_{j}\left[\left(\beta_{m}{ }^{2}-\left(k n_{j}\right)^{2}\right) /\left(\left(k n_{1}\right)^{2}-\beta_{m}{ }^{2}\right)\right]^{1 / 2}
$$

and $j=0,2$ depending on the interface.

These expressions indicate that the form of the waveguiding equation is somewhat different depend. ing on the polarisation of the guided plane-wave: the phase change at the film interfaces depends on whether the interface reflects a transverse electric wave, in which case the wave has an electric field component in the ' $y$ ' direction $\left(E_{y}\right)$ and magnetic field components in the ' $x$ ' and ' $z$ ' directions $\left(H_{x}\right.$ and $H_{z}$ ), or whether the interface interacts with a transverse magnetic wave which has field components $E_{x}$, $E_{z}$ and $H_{y}$.

$$
\begin{array}{ll}
A_{j}=1 & \text { for TE waves } \\
A_{j}=\left(n_{1} / n_{j}\right)^{2} & \text { for TM waves }
\end{array}
$$

The mode numbers ' $m$ ' corresponds to different ray angles $\theta_{m}$ and hence to different field distributions as shown in Figure 1. 


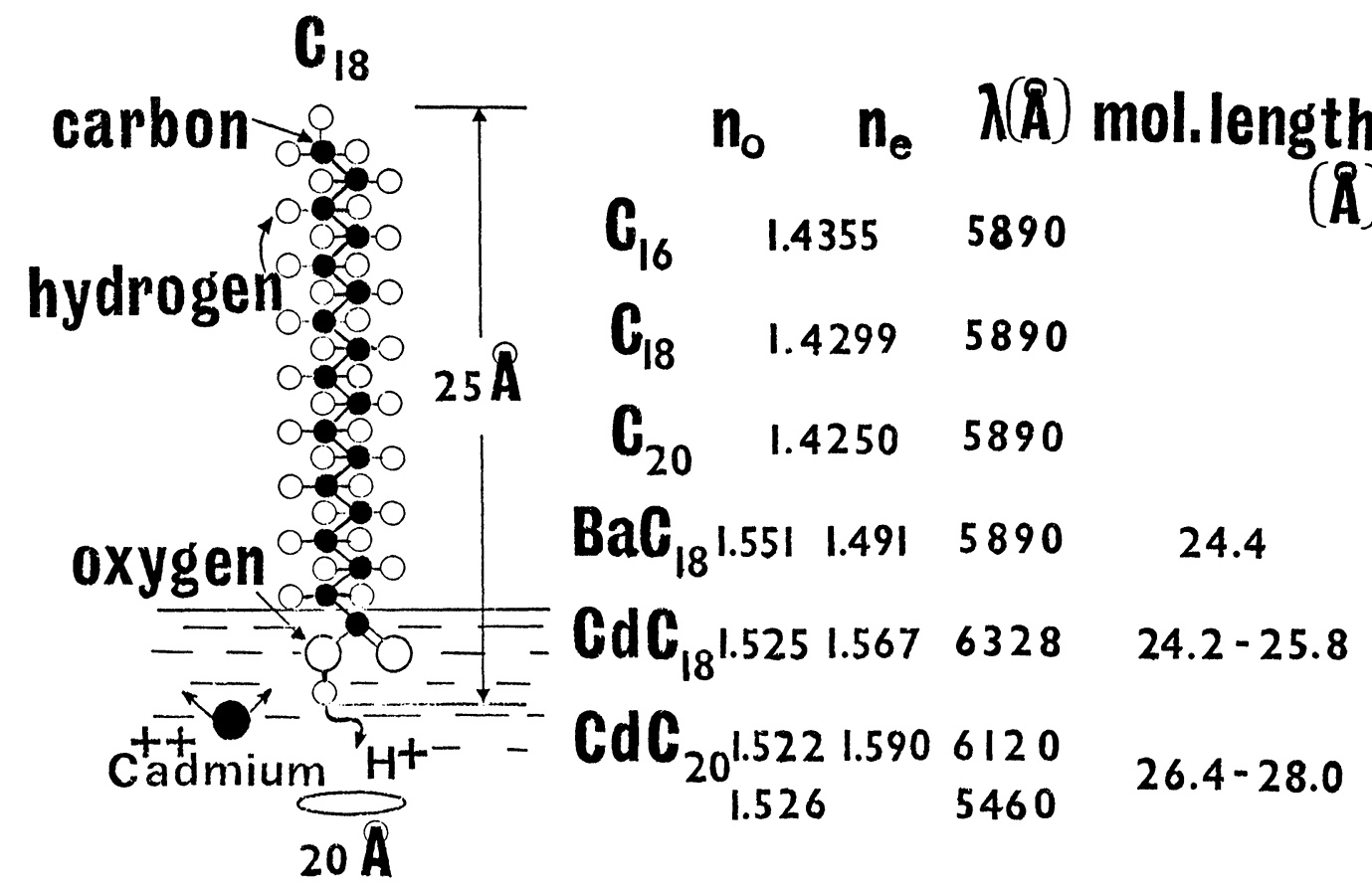

FIGURE 3 Diagram of the structure and properties of some fatty-acid molecules. The molecular length values differ between optical and capacitance measurements.

\section{FILM DEPOSITION}

In this section we briefly review the LangmuirBlodgett method for depositing thin organic films and the type of materials which may be utilized in this process. Figure 3 shows the form of one of the simplest molecular structures suitable for Langmuir film deposition. It consists of $\mathrm{CH}_{2}$ building blocks with a<smiles>O=CO</smiles>

termination at one end and<smiles>C</smiles>

at the other end. The former is hydrophilic and the latter is hydrophobic. This molecule, when placed on a water surface, will float with the hydrophilic termination immersed in the water and the hydrophobic termination in air. The choice of refractive index selection in this form of molecular structure is achieved by selection of an appropriate number of $\mathrm{CH}_{2}$ building blocks or by replacing the $\mathrm{H}^{+}$ions by metallic ions. To satisfy the requirement that the film index should be greater than that of the substrate we employed Cadmium as the substitution ion.

The deposition process is simple and well documented $^{5,7}$. The molecules (Stearic acid) were dissolved in a volatile liquid (e.g. petroleum ether, chloroform) and the solution dispersed on a water surface. The solvent evaporated leaving the molecules partially immersed in the water, and with the long axis randomly oriented (Figure 4a). The molecules were orientated normal to the water-air interface by

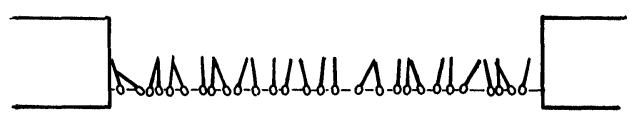

(a) Before Compression

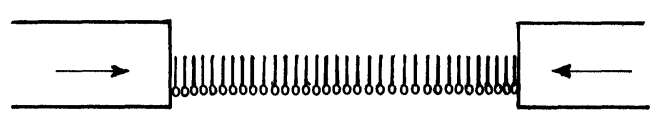

(b) After Compression

FIGURE 4 Diagram of the dispersion of fatty-acid molecules on a water surface. (When a solution of the fattyacid is placed on a water surface the solvent evaporates leaving the molecules dispersed (a). The molecules are aligned by compression (b).) 

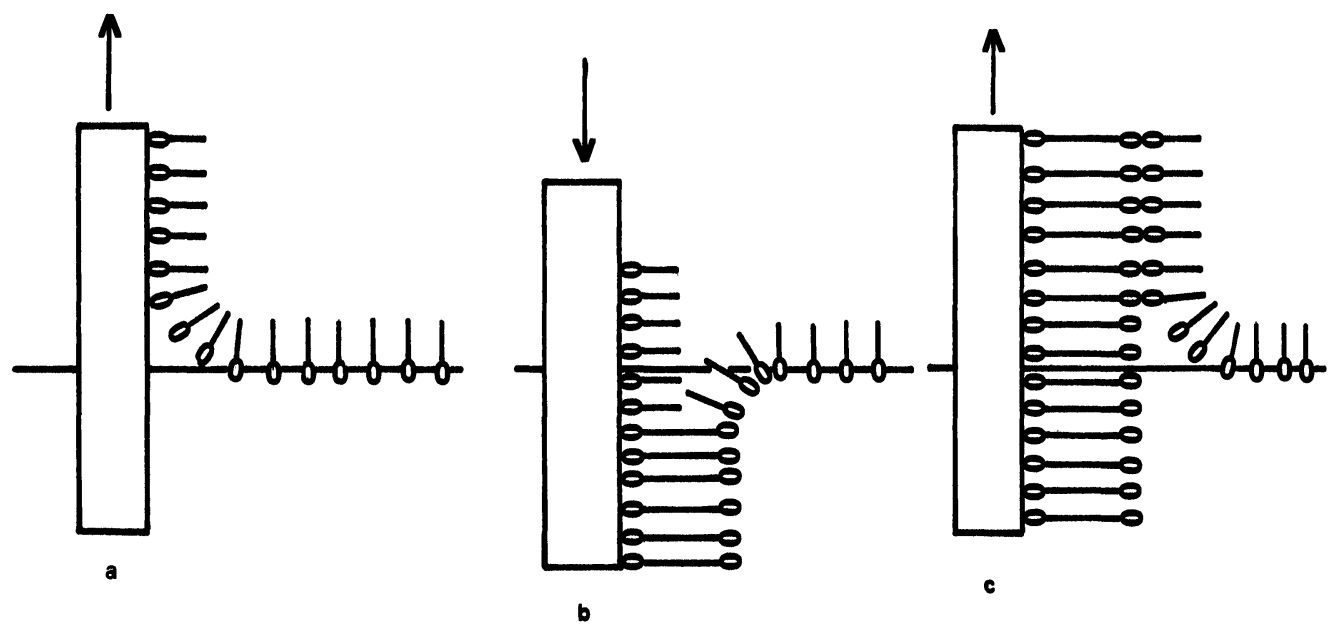

FIGURE 5 Diagrams showing the effect of immersion and removal of a substrate into water with fatty-acid molecules on the surface. (a) Effect of initial removal. (b) Effect of re-immersion. (c) Effect of second removal.

compression with a barrier system as indicated in Figure $4 \mathrm{~b}$. The metallic salt of the molecules was obtained by introducing metallic ions in to the water.

Figure 5a shows a hydrophillic substrate being withdrawn from the water with the hydrophilic termination of the molecular film adhering to the substrate as it is removed. The exposed surface has now become hydrophobic. On the second immersion the substrate will collect another layer but now the hydrophobic termination of both deposited and suspended films will adhere (Figure 5b) - converting the substrate surface again to a hydrophilic surface. The process can be repeated (Figure $5 \mathrm{c}$ ) until the desired number of layers have been deposited.

\section{THE LANGMUIR TANK}

Our system (Figure 6) consisted of a glass tank filled with water to a depth of 5-8 cms. The molecules were dispersed on the water surface to fill the region between the two glass plates and the gravity operated barrier. We used a Wilhemy plate balance to measure the compression force on the molecules, by monitoring the film surface-tension. The tank was covered with a dust cover to keep the immediate ambient dust free. The whole system was placed on an antivibration table in order to prevent film collapse due to vibration. In addition the solution temperature and the $\mathrm{pH}$ were controlled. The slide immersion and withdrawal mechanism consisted of a substrate clamp

$\dagger$ As the molecular film was spread on the liquid surface after the substrate had been totally immersed this would be the first movement of the substrate through the surface. in a vertically mounted gantry, the motion of the substrate being controlled by a linear-motion-drive operated through a reduction gear box. The direction of motion was controlled by reversing micro-switches mounted on the gantry. This mechanism is not shown in the figure.

\section{FACTORS AFFECTING THE HOMOGENEITY AND THE OPTICAL QUALITY OF LANGMUIR FILMS}

In order to obtain high optical quality Langmuir films it was found in preliminary experiments that certain aspects of the deposition process were critical: e.g.

1) a contamination-free sub-phase (water) and purified film material

2) the pressure-relative molecular area relationship

3) the $\mathrm{pH}$ of the sub-phase

4) the temperature of the sub-phase

5) the deposition rate and pressure variation occurring in the suspended monolayer during deposition.

\subsection{Contamination of the Tank, the Water, or the Fatty Acid Molecules}

Any alien molecules, either organic or inorganic, found in the sub-phase as contaminants can become incorporated into the film structure. Their presence in the structure may modify the nature of the polar/ non-polar orientation of the successive layers and 


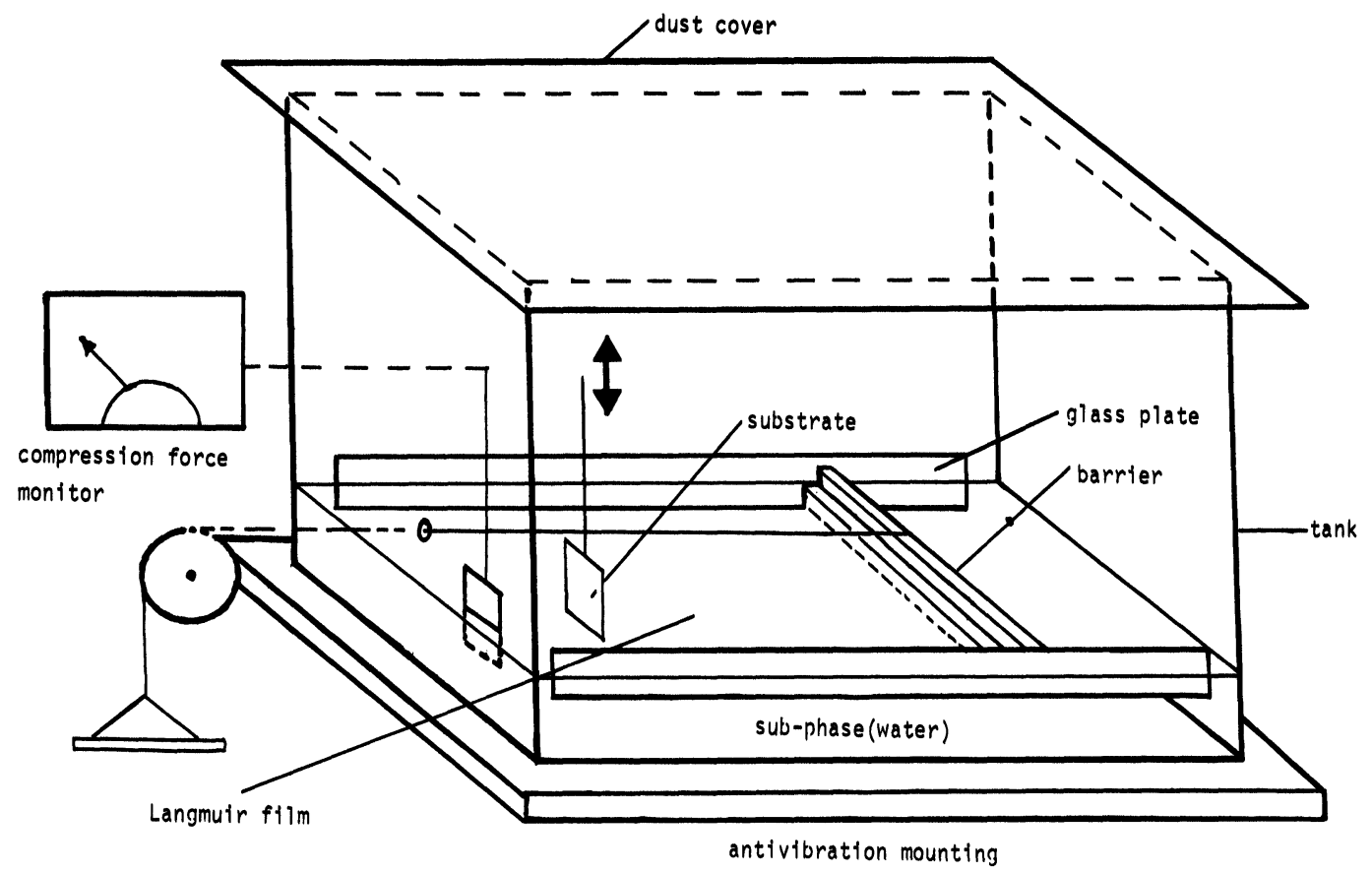

FIGURE 6 Langmuir film deposition apparatus.

may therefore result in wetted areas being occluded into the multilayer film. The occluded water may cause splitting of the films or pin holes, or the alien molecules may act as absorption or scattering centres in the waveguiding film. It is therefore important that the tank should be scrupulously clean, the film material extremely pure, and the subphase water distilled several times to exclude contaminants.

\subsection{Pressure-Relative Area Relationship}

This relationship is illustrated in Figure 7. At low pressure the molecules are loosely packed and occupy a large area; i.e. they are separated by an exposed water surface. If the molecules remain loosely packed, the layers deposited onto the substrate will contain molecular vacancies often filled with water. On the other hand, if the molecules are too tightly packed the film on the subphase could collapse resulting in agglomerated regions which are several layers thick. The subphase monolayer is therefore compressed to the point at which the molecular area remains effectively constant for a large change in pressure, thus ensuring that small changes in pressure caused by the immersion and withdrawal of the substrate do not cause the film to break up or fold over.

\subsection{The Sub-phase $p H$}

The degree of ionization of the fatty acid in the subphase depends on the $\mathrm{pH}$ of the solution ${ }^{8}$. A strongly basic subphase will cause enhanced ionization and hence increase the fatty acid to salt conversion: an increased film refractive index results from the enhanced molecular polarisability produced by the metal ions included in the film. In addition a relatively high $\mathrm{pH}$ will improve the film/substrate adhesion. There are however some disadvantages; the monolayer on the water surface may become less stable, the solubility of the molecules in the subphase may increase, and also metal hydroxides can precipitate. These factors will obviously cause the film defect level to increase. We therefore optimised the process, having found both refractive index and adhesion satisfactory for films deposited at $\mathrm{pH}$ values between 5 and 7 . These relatively low $\mathrm{pH}$ values produced adequate quality film-deposition on most metal oxide substrates (e.g. $\mathrm{Al}_{2} \mathrm{O}_{3}, \mathrm{MgO}$ ) but were not satisfactory in the case of fused quartz substrates. We therefore treated quartz glass substrates by immersion in an alkali solution to modify the surface layer. The method has been described by Bucher et $\mathrm{al}^{9}$. 


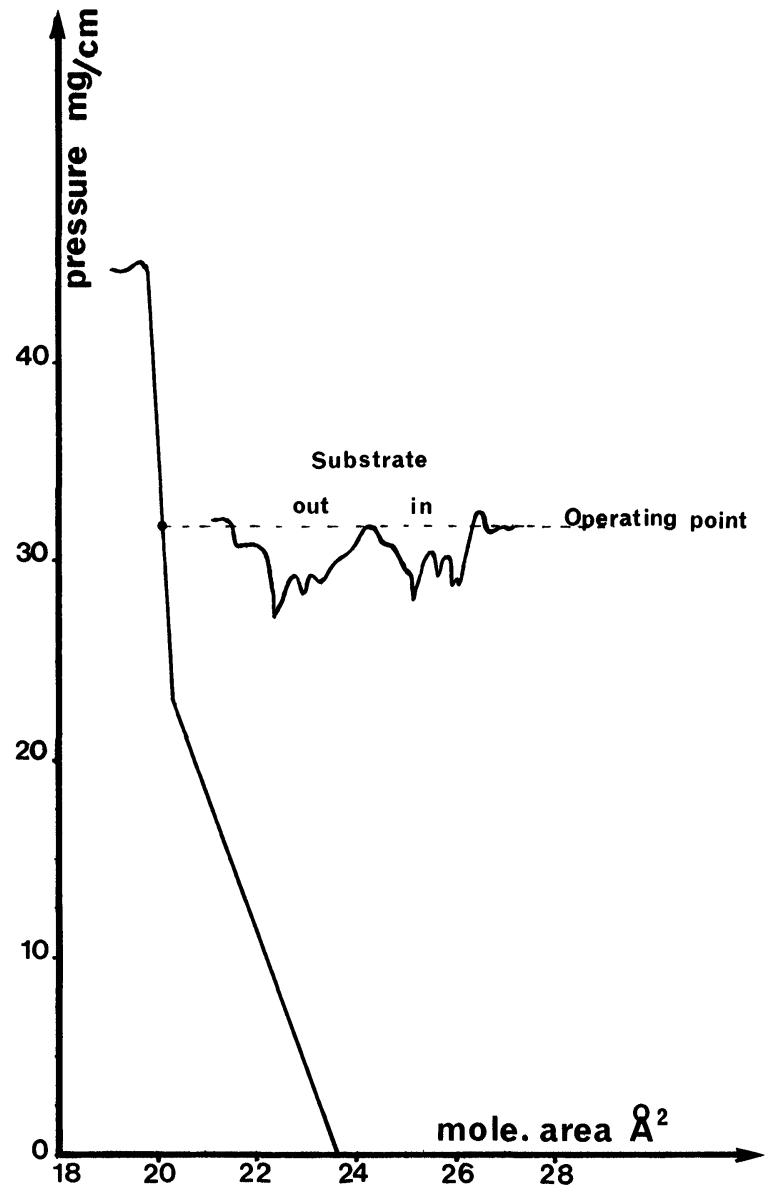

FIGURE 7 Monolayer compression pressure vs. surface coverage showing ideal operating region.

\subsection{The Temperature of the Solution}

The mono-layer properties on the subphase will also depend on the solution temperature ${ }^{8}$. The condensation of the molecular layers to form a monolayer on the water surface is made easier by decreasing the temperature. The low temperatures may, however, increase the viscosity of the monolayer thus making it brittle. On the other hand high temperatures can cause the films to expand and ultimately may result in thermal forces overcoming the rather weak Van der Waal's binding forces which hold the molecules together. This may enhance the molecule solubility and will certainly make the layer unstable. We have noted that the temperature range $19-22^{\circ} \mathrm{C}$ is suitable for Cadmium Stearate film deposition.

\subsection{The Deposition Rate and Pressure Variation}

In order to obtain good quality Langmuir-films it is important that the initial layers are well formed, as faults in these layers may be replicated in later layers. To ensure good adhesion the initial layers were deposited onto the substrate at low immersion and withdrawal rates $\sim 3 \mathrm{~mm} / \mathrm{min}$. When a few layers have been formed the rate may be increased to a higher value, in our case $24-30 \mathrm{~mm} / \mathrm{min}$. We have observed pressure variations in the mono-layer on the water surface during deposition; as we have already suggested the film must be maintained in the condensed phase so that the molecules are not loosely, or too tightly, packed. In Figure 7 we have plotted the pressure change during immersion and withdrawal of the substrate. The operating point $(\sim 30 \mathrm{mg} / \mathrm{cm})$ is so chosen that the pressure variation is maintained between $25-35 \mathrm{mg} / \mathrm{cm}$. Clearly the substrate immersion and withdrawal rate affects the amplitude of these perturbations - and it is this factor which sets the maximum substrate velocity.

\section{THE FILM QUALITY}

The film defects usually show up in the form of cracks, holes and agglomerations. The major reasons for these defects have already been discussed. Figure 8 shows two optical micrographs one with many defects (Figure $8 \mathrm{~b}$ ) and the other with a significantly reduced level (Figure 8a). Films of the type shown in Figure $8 \mathrm{~b}$ are not suitable for low loss optical waveguide applications, as the defects scatter light from the waveguide, and also scatter energy between the propagable modes of the structure. This effect will obviously result in high optical attenuation. The film in Figure 8a, with less defects, has much lower scattering losses and is therefore more useful as an optical waveguide.

\section{CHARACTERISATION OF LANGMUIR FILM WAVEGUIDE}

Initially we deposited Langmuir films of thickness 80-120 layers. These films could not be used as asymmetrical optical waveguides as the minimum cutoff thickness for guiding was much larger than the deposited layer thicknesses, i.e. we could not achieve a thickness (W) such as to satisfy Eq. (2) for $m=0$. In order to obtain guiding it was therefore necessary to use these films as symmetrical waveguides. It may be shown, by making $n_{0}=n_{2}$ in the auxilliary Eq. (3) and substituting in Eq. (2), that a symmetrical waveguide has a minimum cut-off thickness of zero for the $m=0$ mode. In other words we 

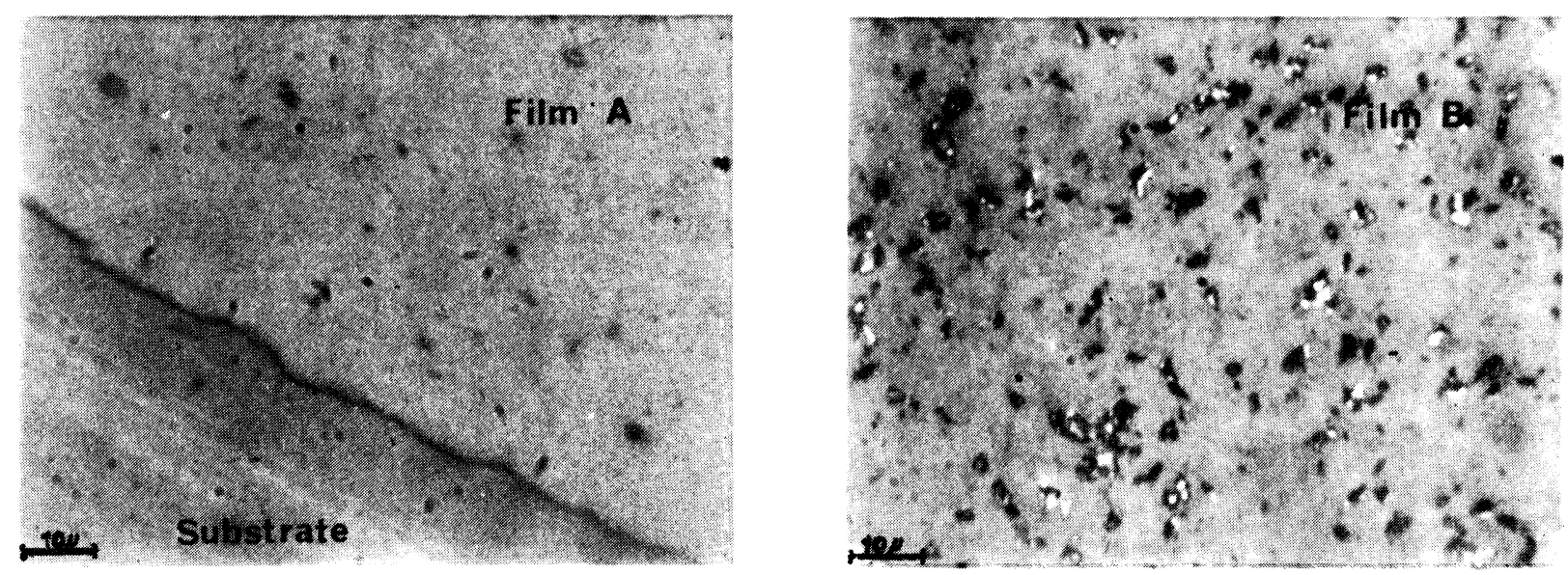

FIGURE 8 Optical micrographs of dielectric films (a) With low defect level (b) With high defect level.

can have a symmetrical guide of infinitely small thickness and still exhibit wave-guiding. The Langmuir film structures were made symmetrical by placing a chemically non-reactive liquid on top of the film so that the liquid index was equal to the substrate index (Figure 9).

To couple light into the Langmuir films we have used a symmetrical prism ${ }^{10}$. For light coupling to be satisfactory the prism index must be much larger than

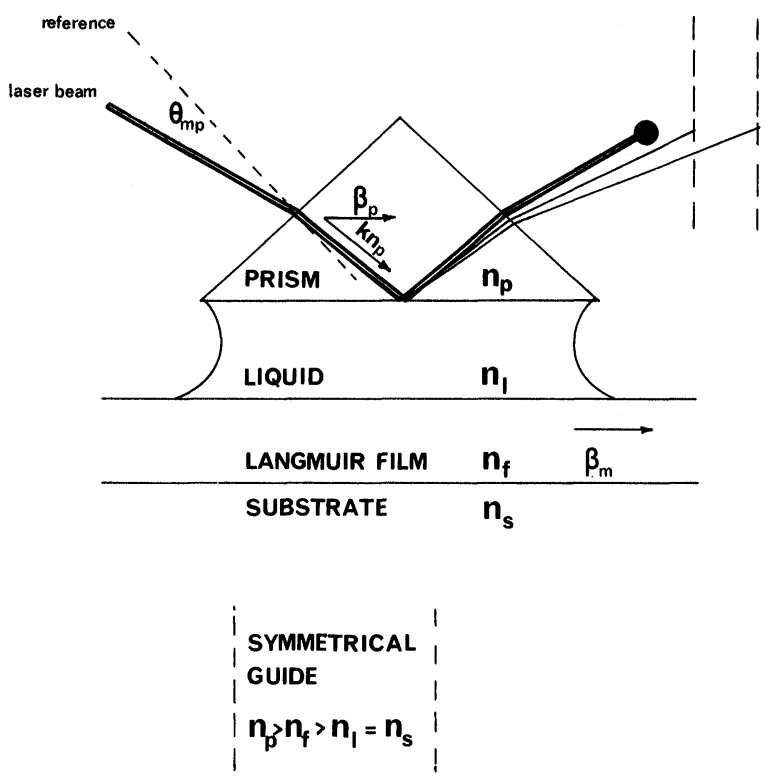

FIGURE 9 Diagram of a symmetrical Langmuir waveguide and prism-coupler. (For the laser beam to be coupled into the waveguide, the film-plane component of the beam velocity in the prism $\left(\beta_{p}\right)$ must be equal to the velocity of propagation of the excited mode $\left.\left(\beta_{m}\right)\right)$. the film indices. The high index prism is required to match the laser beam velocity to the guided wave velocities. The light is reflected at the base of the prism (Figure 9) and coupling is achieved by optical tunneling when the evanescent field in the prismfilm gap travels at the same velocity as the mode to be excited. This condition is only satisfied for a particular angular position $\left(\theta_{m p}\right)$ of the incident beam of light. Similarly light is coupled out and may be projected onto a screen. The guided light may be distinguished from the light reflected from the base of the prism by the appearance of 'mode lines' at clearly separate angular positions. The angular positions $\theta_{m p}$, at which the mode lines are excited may be measured with respect to a defined reference and, by solving the waveguide Eq. (2) simultaneously for several modes using the values of $\beta_{m / k}$ derived from the $\theta_{m p}$ values, the film index and thickness may be determined. Note that Langmuir films are soft and that the liquid placed between the prism and the film also reduces deformation and damage by the prism.

Langmuir films are in general anisotropic and the isotropic equations already discussed must be modified in order to take both $o$-ray and $e$-ray index into consideration ${ }^{11}$. Several relatively thin (few tens of layers) Langmuir films were deposited under the following conditions:

Temperature
Metal ion concentration
Water
Substrate-fused quartz
Deposition rate

$19-22^{\circ} \mathrm{C}$ $2 \times 10^{-3} \mathrm{Mol} . \mathrm{CdCl}_{2}$ Distilled ( $x 4)$

Alkali soak $3 \mathrm{~mm} / \mathrm{min}$ initial $24 \mathrm{~mm} / \mathrm{min}$ after few layers 
TABLE I

Results from optical waveguide measurements.

\begin{tabular}{|c|c|c|c|c|c|c|c|}
\hline \multirow[t]{2}{*}{ Sample } & \multirow[t]{2}{*}{$\mathrm{pH}$} & \multicolumn{3}{|c|}{$\begin{array}{l}\text { Refractive indices and molecular length } \\
\text { obtained from optical measurements only. }\end{array}$} & \multicolumn{3}{|c|}{$\begin{array}{l}\text { Refractive indices and Molecular length } \\
\text { obtained from optical/mechanical thickness } \\
\text { probe measurements. }\end{array}$} \\
\hline & & mol. length A & o-ray index & e-ray index & mol. length $A$ & o-ray index & e-ray index \\
\hline 1 & 5.3 & $\begin{array}{l}24.2 \pm 0.3 \\
24.5 \pm 0.8\end{array}$ & $1.511 \pm 0.001$ & $1.548 \pm 0.002$ & $25.2 \pm 0.6$ & $1.509 \pm 0.001$ & $1.545 \pm 0.002$ \\
\hline 2 & 5.6 & $\begin{array}{l}23.8 \pm 1.3 \\
25.0 \pm 6.3\end{array}$ & $1.526 \pm 0.003$ & $1.568 \pm 0.017$ & $24.1 \pm 0.3$ & $1.526 \pm 0.001$ & $1.569 \pm 0.002$ \\
\hline
\end{tabular}

and then characterised by probing the symmetrical waveguide structure with a guided wa ve at heliumneon laser wavelength ( 0.63 microns). A modified form of the waveguide equation, which included index anisotropy, was solved to produce both 0 -ray and $e$-ray refractive index values. These values were calculated from $\mathrm{TE}_{0}$ and $\mathrm{TM}_{0}$ mode measurement. $A$ semi-independent check on the reliability of these measurements was obtained by measuring one of the variables, the thickness $\mathrm{W}$, by means of a mechanical film-thickness probe; the results are compared in Table I.

We observed that the refractive indices at low $\mathrm{pH}$ are smaller than the value obtained at higher $\mathrm{pH}$. This suggests that the metal ion $\left(\mathrm{Cd}^{++}\right)$concentration in film is dependent on the $\mathrm{pH}$ value of the solution. An increased metal ion concentration in the film will enhance the polarisability of the molecular structure and increase the refractive index. However there are other effects ${ }^{12}$ resulting from $\mathrm{pH}$ adjustment; typically expansion of the molecular areas, as a

TABLE II

Characteristics of asymmetrical waveguides

Guide A

No. of layers

Modes

Average loss

Propagation path length

281

$\mathrm{TE}_{0}, \mathrm{TM}_{0}$ $6.2 \mathrm{~dB} / \mathrm{cm}$ TM $5.1 \mathrm{~dB} / \mathrm{cm} \mathrm{TE}$ $20 \mathrm{~mm}^{*}$

Guide B

No. of layers

Modes

Average loss

Propagation path length

221
$\mathrm{TM}_{0}$
$3.6 \mathrm{~dB} / \mathrm{cm} \mathrm{TM}$
$20 \mathrm{~mm} *$

*The propagation path length was restricted only by the size of the substrate. result of the reduced inter-molecular binding forces, and which effectively reduce the refractive index. Thus the experimental values of $n_{0}$ and $n_{e}$, as a function of $\mathrm{pH}$, appear to be the result of two competing mechanisms.

It was also observed that the uncertainty in the results in the second set ( $\mathrm{pH} \mathrm{5.6)}$ is much larger than the first set ( $\mathrm{pH} \mathrm{5.3).} \mathrm{We} \mathrm{believe} \mathrm{that} \mathrm{this} \mathrm{is} \mathrm{a} \mathrm{result}$ of the poorer quality of the films in the second set, a belief which has been confirmed by optical examination which revealed a larger number of pinhole defects.

\section{LANGMUIR FILMS AS ASYMMETRICAL WAVEGUIDES}

In order to obtain asymmetrical waveguiding in Langmuir films we deposited larger thickness Cadmium Stearate films under similar conditions to those outlined. These films were studied as planarwaveguides by coupling $\mathrm{He}-\mathrm{Ne}$ ( 0.63 microns) laser light into them by means of a right-angled prism; the attenuation characteristics, along various lengths of propagation path, were examined both by collecting the light scattered by the defects along the propagating streak (using a photo-diode) and also by an output coupler prism ${ }^{13}$ (Table II).

The film A, of 281 layers, was more attenuative than film B (221 layers). The reasons for this are thought to be the higher defect level in film $A$ compared to that of film B. However we observed during the measurements that the film attenuation varied considerably along the propagation path and in places was less than $1 \mathrm{~dB} / \mathrm{cm}$ - which encourages the belief that further process improvements may reduce average losses to below this value. 


\section{LANGMUIR FILMS AS CLADDINGS ON OPTICAL WAVEGUIDES}

We have looked at the use of Langmuir films for "fine tuning" the mode characteristics of prefabricated waveguides. When an optical waveguide is perturbed, by depositing a cladding over the guide-air interface, the propagating mode electric-field distribution is extended into the cladding (Figure 10). In this case, the cladding will carry a significantly larger amount of energy compared to the air superstrate case. The result will be an increase in the normalised propagation constant of the propagating mode. It is worthwhile mentioning at this point that there are two possible configurations for the superstrate; (i) the cladding refractive index $>\beta / k$ for the propagating mode and (ii) the cladding index is $<\beta / k$. When the

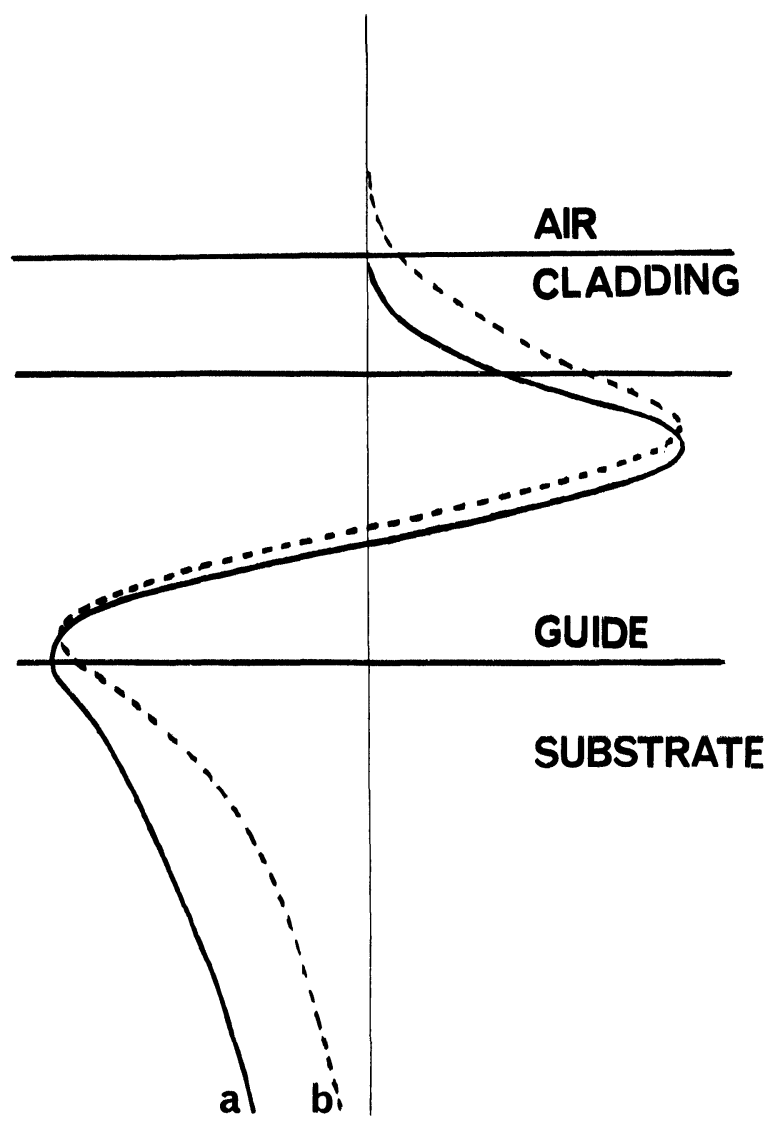

FIGURE 10 Diagram showing the effect of cladding on propagation in an optical waveguide. (The curve ' $a$ ' is the electric-field distribution of the mode $T E$, of the unperturbed waveguide. The curve ' $b$ ' is the field distribution of the mode $T E_{1}$ when perturbed by a cladding).

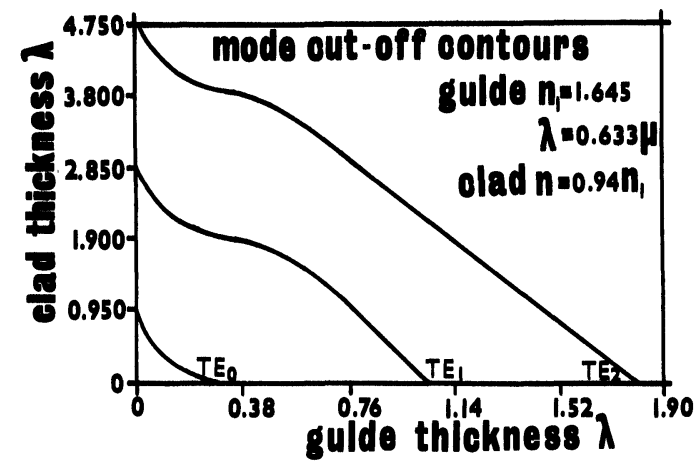

FIGURE 11 Mode cut-off contours for cladded waveguides.

cladding index is greater than $\beta / k$, the electric-field will vary sinusoidally in the cladding in the direction normal to the guiding film. In the case of the lower cladding index, the electric-field will decay exponent. ially in to the cladding. The sinusoidal electric-field in the cladding will carry rather more of the guided energy than the evanescent field and hence will produce a much larger modification to the propagation constant.

The waveguide plus cladding structure is essentially a two-layer waveguide. For structures of this type, we have calculated mode cut-off contours and experimentally verified that additional modes can be introduced into prefabricated waveguides by adding superstrate layers (Figure 11). However the superstrate thickness is then rather larger than the waveguide thickness, so that this does not appear to a practical application for Langmuir films. The most attractive feature of Langmuir films being their controlled thickness and refractive index, the device format which utilizes these properties most advantageously appears to be a prefabricated wave.

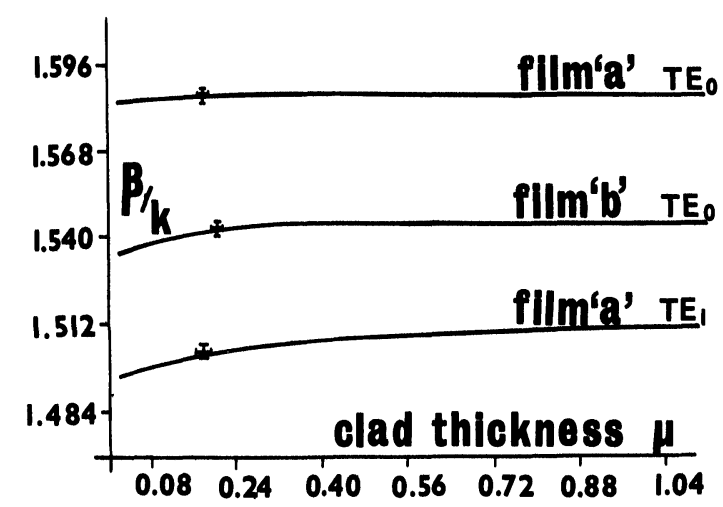

FIGURE 12 Normalised propagation constant vs, cladding thickness. 


\begin{tabular}{ll}
\hline FILM GUIDE ' $b$ ' & $n=1.63 \quad \begin{array}{c}t=0.262 \mu \\
\beta_{T_{0}}=1.5046\end{array}$ \\
\hline SPACER & $n=1.457 \quad t=1.2 \mu$
\end{tabular}

\begin{aligned} \hline FILM GUIDE 'a' $\quad n=1.63 \quad t=0.231 \mu \\ \beta_{T E_{0}}=1.5247\end{aligned}$

SUBSTRATE

$n=1.457$

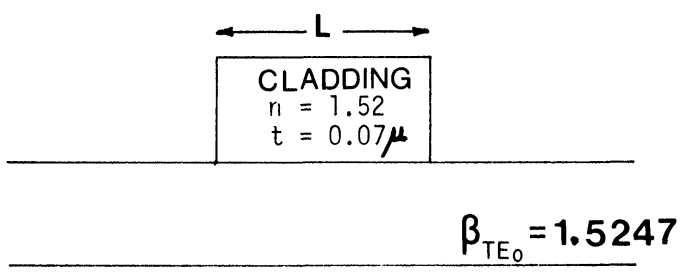

$$
\beta_{T E_{0}}=1.5247
$$

FIGURE 13 Diagram showing a multilayer guide structure.

guide with relatively small $\beta / k$ perturbation induced by applying a few monolayers.

The Figure 12 shows curves of normalised propagation constant against the cladding thickness derived from straightforward dielectric slab-waveguide theory. The experimental points are plotted as shown. The error bars indicate the tolerance in measurement of the propagation constant and the uncertainty in thickness measurement. The precision to which the mode propagation constant can be adjusted is apparent from the agreement between the measured values and the waveguide equation prediction.

\section{APPLICATIONS}

The basis for design of integrated optical components is an accurate knowledge of the refractive index of the waveguiding film material, or the capability to change the index to desired value. In addition, the designer needs a fabrication process that will deposit a film of precisely determined thickness. Langmuir films meet these requirements, and, perhaps more importantly, enable waveguides formed in inorganic materials to be adjusted in a post-fabrication operation.
There are many potential applications for propagation constant adjustment, one of which is to bring two optical waveguides into synchronism. Figure 13 shows the section through two planar waveguides separated by a spacer layer. The two waveguides have modes of different wave velocities - the velocity in waveguide ' $b$ ' being lower than that in ' $a$ '. In order to obtain velocity synchronism between the two guides a cladding may be added onto the guide ' $b$ '; the effect on the guide 'a' will be negligible as a result of the spacer layer. Thus the wave velocities in the two guides may be made equal by depositing a precalculated number of Langmuir film layers on to guide ' $b$ ': the guided mode in ' $b$ ' is perturbed and $\beta / k$ is increased from 1.5046 to 1.5247 i.e. equal to that of guide ' $a$ '. Under these conditions the energy in one guide will transfer into the other guide via the overlapping evanescent fields, and the energy will periodically exchange between the two waveguides. The oscillatory transfer of energy may be prevented by terminating the length ' $L$ ' of the cladding so that the energy is retained in the desired guide after a single transfer. This type of transfer device is often termed a waveguide coupler.

\section{CONCLUSIONS}

In this brief review of our work on Langmuir films we have indicated how oriented organic dielectric films can be deposited in multilayers to form optical waveguides, how the refractive index and molecular thickness can be estimated by probing the films with a guided wave and how such layers can be utilized to modify the propagation characteristics of prefabricated guiding structures.

Clearly the most attractive feature of these films for integrated optics applications is the capability to control the waveguide propagation constants via the precise refractive index and film thickness properties of the molecules. The optical attenuation of the films along several centimeters of propagation path is also sufficiently small for experimental devices. We foresee that the average attenuation might be reduced to less than our lowest values to date (circa $1 \mathrm{~dB} / \mathrm{cm}$ ) by using modified materials - perhaps polymerisable molecules. In addition to reducing the number of defects in the film, polymerisation may produce harder films which would be less susceptible to damage by the prism-coupler.

To speculate further, we also believe that some of the other properties of this process may prove important in the future - e.g. the incorporation of 
metal ions and dye molecules into Langmuir films is a simple procedure and suggests the fabrication of a thin-film laser using the Langmuir films as the inert matrix.

\section{ACKNOWLEDGMENTS}

This work is supported by Imperial Chemical Industries Ltd. under the aegis of a Joint Research Scheme. We are grateful to W. A. Barlow, R. A. Hann and J. A. Finney of the I.C.I. Corporate Laboratory, for continuing support and inspiration.

\section{REFERENCES}

1. R. Ulrich, "Thin dielectric films for integrated optics", J. Vac. Sci. Technol., 11 (1974) pp. 156-162.

2. C. W. Pitt, F. R. Gfeller, and R. J. Stevens, "Sputtered thin films for integrated optical components", Thin Solid Films, 26 (1975) pp. 25-51.

3. R. Ulrich and H. P. Weber, "Solution-deposited thin films as passive and active light guides", Applied Optics, 11 (1972) pp. 428-434.

4. I. P. Kaminnow, and J. R. Carruthers, "Optical waveguiding layers in $\mathrm{LiNbO}_{3}$ and $\mathrm{LiTaO}_{3}$ ", Appl. Phys. Lett., 22 (1973) p. 326.
5. B. K. Blodgett, and I. Langmuir, "Built-up films of barium stearate and their optical properties", Phy. Rev., 51 (1937) pp. 964-981.

6. P. K. Tien, "Light waves in thin films and integrated optics", Applied Optics, 10 (1971) pp. 2395-2413.

7. E. Wolf, (Ed), Progress in Optics. Vol XII, published by North-Holland/American Elsevier, 1974.

8. G. L. Gaines, Insoluble Monolayers at Liquid Gas Interface, published by Interscience, 1966.

9. H. Bucher, et al. "The detection of the separation of monolayer dyestuff - arachidic acid films by the energy transfer method", Zeitschrift für Physikalische Chemie, New Series V65 (1969) pp. 152-169.

10. P. K. Tien, and R. Ulrich, "Theory of the prism-film coupler and thin film light guides", J. Opt. Soc. Am., 60 (1970) pp. 1325-1338.

11. L. M. Walpita, and C. W. Pitt, "Measurement of Langmuir film properties by optical waveguide probe", Elec. Lett., to be published.

12. J. Bagg, et al. "Composition of stearic acid monolayers from calcium-containing substrates", J. Am. Chem. Soc., 86, 14 (1964) pp. 2759-2763.

13. C. W. Pitt, and L. M. Walpita, "Optical waveguiding in Langmuir films", Elec. Lett., 12 (1976) pp. 479-481.

14. V. K. Srivastava, Physics of Thin Films, Vol. 7 (Eds. G. Hass, M. H. Francombe, R. E. Hoffman), p. 311, 1973).

15. V. K. Agarwal, Electrocomponent Science \& Technology, 2 p. 1, (1975). 

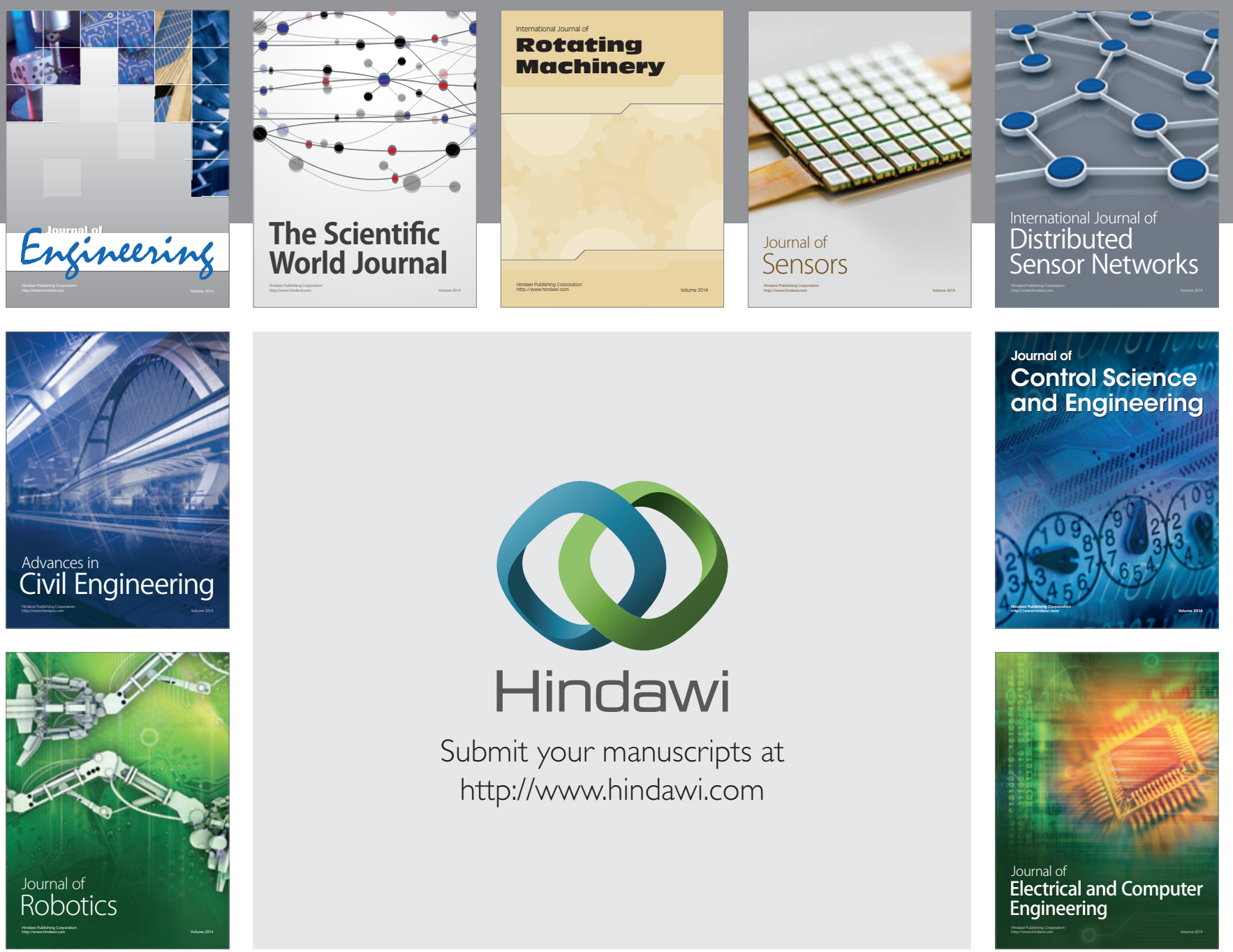

Submit your manuscripts at

http://www.hindawi.com
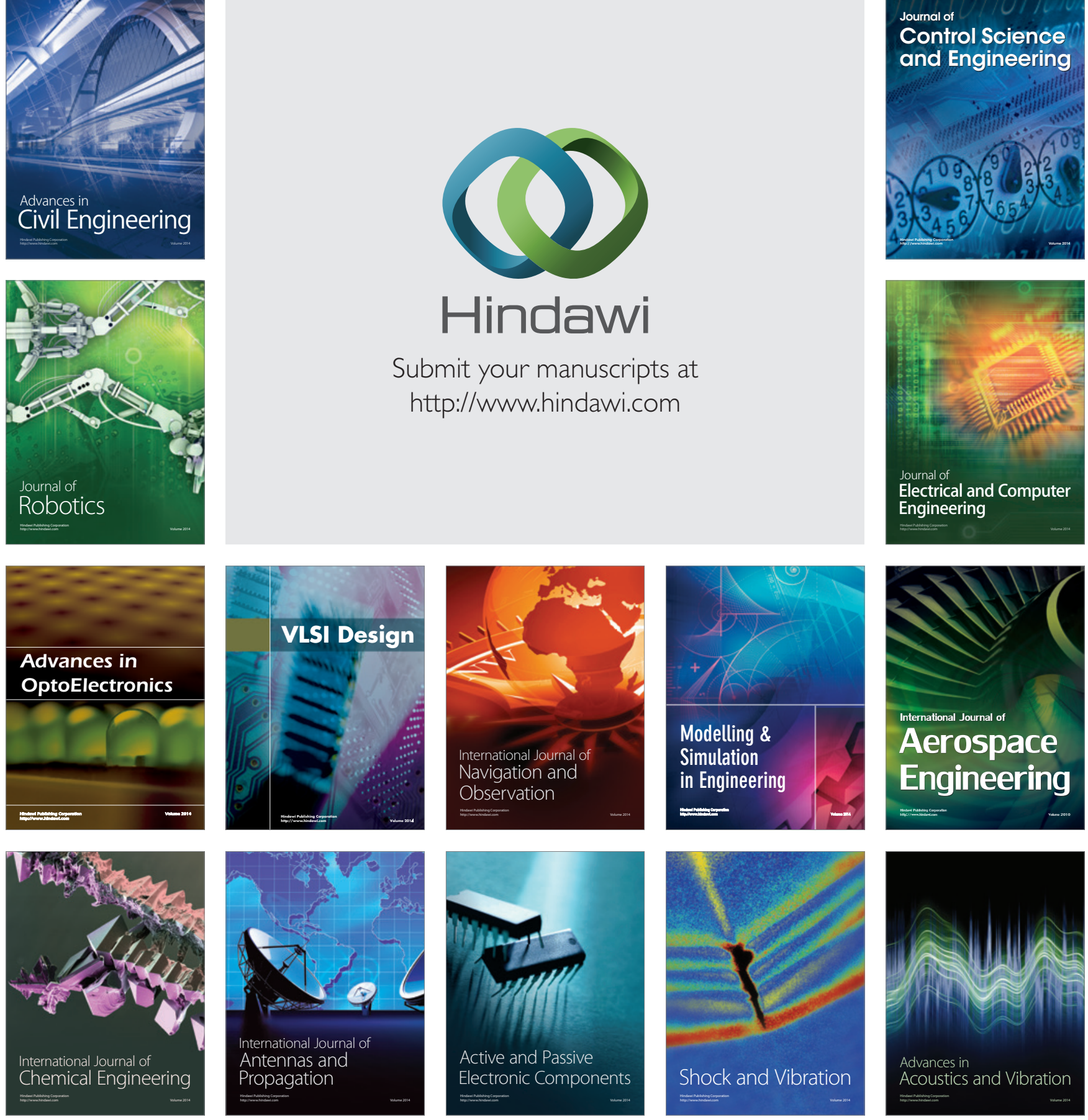